\title{
Genetic Introgression Between Different Groups Reveals the Differential Process of Asian cultivated Rice
}

Hao Gong ( $\nabla$ mygonghao@163.com )

Huizhou University

Bin Han

Chinese Academy of Sciences

\section{Research Article}

Keywords: Genetic introgression, admixture mapping, gene interchange, QTL mapping.

Posted Date: December 16th, 2021

DOI: https://doi.org/10.21203/rs.3.rs-1168682/v1

License: (c) (1) This work is licensed under a Creative Commons Attribution 4.0 International License.

Read Full License 


\section{Abstract}

Genetic introgression plays an important role in the domestication of crops. The Asian cultivate rice consists of two major subspecies, they are indica and japonica. There are already many reports about existence of genetic introgression between the two subspecies. However, those studies often use few limited markers to characterize the genetic introgression that exists in some specific small populations. In this study we use the genome wide variation data of Asia cultivated rice to investigate their genetic introgression on the whole genome level. We detect a total of 13 significantly high introgression loci between the tropical japonica and indica population. Two different methods are used to identify the genetic introgression regions. For most of the detected introgression regions they generally get consistent results. Some previous known introgression genes are detected in the identified introgression loci, such as heat resistance gene TT1 and GLW7. The biological functions for these genetic introgression regions are annotated by the published QTL mapping results. We find that genetic introgression plays an important role in both the determination of the phenotype and the domestication process of different groups. Our study also provides useful information and resources for the study of rice gene function and domestication process.

\section{Introduction}

Genetic introgression is the process of integration the alleles from one species to another gene pool. Many generations of hybridization and back-crossing are needed to make the introgression alleles stable. Different from the simple hybridization process genetic introgression is a long process (Zhang et al., 2016, Durand et al., 2011 Chen et al., 2020 Civan et al., 2018). The simple hybridization process is an equal mixture of the two parent genomes in the F1 population. However, genetic introgression is the mixture of genetic alleles of many different accessions. Genetic introgression can be divided into the human mediated process and nature process. The breeding process of introducing the segments that control favorable traits from one population to another is an evident process of human mediated introgression (Ando et al., 2008). Human mediated introgression is prevalent in the animal and crop domestication process. Nature introgression often happens in the process of adapting to the local environment (Huerta et al., 2014, Racimo et al., 2015, Lin et al., 2020 Santos et al., 2018 Wedger et al., 2019). For examples, recent reports show that the early Tibetans acquire the allele to improve the binding of hemoglobin to the oxygen from the Denisovan like ancient human population (Huerta et al., 2014). There are reports of genetic introgression in the cultivated maize from the wild maize in the highland of mexico. They discover wide spread wild maize genome component in the cultivated maize genome (Hufford et al., 2013). By transmitting the introgression segment of wild maize to the cultivated maize, they show that these segments can improve the plant height and pigment extension. In this study we try to characterize the genetic introgression within the subgroups of the cultivated rice using the whole genome variation data of cultivated rice. Two different methods are used to detect the genetic introgression within the cultivated rice. The first one is phylogenetic tree method (Huson et al., 2007, Huson et al., 2012). The second one is D-static method (Durand et al., 2011, Zhang et al., 2016). Different 
from the phylogenetic tree method, D-static method can calibrate the disturbance of the same domestication origin of cultivated rice in the detection of introgression segments by using the genetic variants in the ancient outgroup as the background. The two detection methods get similar results in the introgression regions within the cultivated rice.

To analyze the biological function of these introgression segments in cultivated rice, we use QTL mapping results of a hybrid population constructed with indica and japonica to annotate these regions. Here we use the genome component matrix (Alexander et al., 2009) of the cultivated rice population to conduct admixture mapping to detect the phenotype related introgression regions. Many previous reported phenotype related genes are found to be located in the introgression region. Our study systemically analyzes the genetic introgression within the cultivated rice. By using admixture mapping and QTL mapping results the biological functions for these introgression regions are also investigated.

\section{Results}

\section{Primary study of genetic introgression within the cultivated rice with phylogenetic tree method}

Many previous studies have shown that the domestication of Asian cultivated rice is single origin (Huang et al., 2012, Molina et al., 2011). The wild rice was first domesticated to the ancient japonica. One part of ancient japonica hybridizes with the local wild rice in the south Asia to form the indica subgroup. Another ancient japonica group is domesticated to the modern japonica group. The japonica has a wide distribution in East Asia. It's divided into the temperate japonica and tropical japonica subgroups. Here we analyze the genetic introgression within the Asian cultivated rice groups. However, our study mainly focuses on the genetic introgression between the tropical japonica and indica population. Though the tropical japonica clusters with the temperate japonica in the whole genome phylogenetic tree analysis, the tropical japonica subgroup is similar to the indica subgroup on some phenotypes. For example, heat resistance, grain shape and plant height.

By summarizing the introgression accessions across the whole genome, we construct the map of genetic introgression for the different groups. The introgression regions are found to occupy only a small part of the whole genome (3\%). However, some blocks in the genome are enriched with genetic introgression. There is genetic introgression in nearly all accession in a few blocks (Figure 1). As it's difficult to get high genotyping accuracy in the high repeat sequence of the genome, it may disturb the construction of neighbor joining tree and the genetic introgression detection process. By comparing the genetic introgression level in the high repeat regions with that of the whole genome level, we find no significant of enrichment of introgression in high repeat sequence of rice genome.

To get a clear view of genetic introgression level within the cultivate rice we summarize the introgression accession number on each block. We find that there are some regions which process high introgression level from the indica subgroup to tropical japonica subgroup (Supplementary Figure 1). However, we 
detect few introgression accessions from the tropical japonica group to the indica group in the same introgression regions. As we have characterized the introgression level within different subgroups of cultivated rice (Figure 2), we also check the correlation of the introgression accession number between the different groups in the whole genome level. Interestingly, no significant correlation of the introgression is found between the different groups (Figure 3). For the high introgression regions in different groups there are no consistent regions except one in the centromere of chromosome 5 . So the direction of introgression within the different groups is unidirectional. As previous studies have detected some selective sweep regions between the cultivated rice and wild rice, we find overlap of the selective sweep regions with our detected high introgression regions (Figure 2). Besides human mediated selection, genetic introgression may also play an important role in the domestication of the cultivated rice. Some highly introgression regions that we detect between different rice subgroups, which don't overlap with the selective sweep region, may control other important traits that are not under high selection pressure. Besides overlapping of the introgression with the selective sweep regions, we find that there is great variation in the genetic introgression between different groups. The tropical japonica and temperate japonica are both domestication from the ancient japonica. The two subgroups both exist high genetic distance with the indica group and cluster together in the whole genome phylogenetic tree analysis. However, we find significantly more introgression in the direction from indica to the tropical japonica than that from indica to the temperate japonica in the genetic introgression analysis (chi-square test, $P$-value $<$ $0.01)$.

Using the permutation test results as the background we detect a total of 13 regions that contains significantly high genetic introgression in the whole genome level from indica to tropical japonica. Some previous reported introgression genes are found to be located in our detected high introgression loci. For example, there are TT1 GS3, GLW7 (Li et al., 2015, Fan et al., 2006, Si et al., 2016). They are located in the high introgression region. TT1 is a heat resistant gene, which is found to be introduced to the tropical japonica from the indica by genetic introgression (Figure S1) (Wang et al., 2014, Li et al., 2015). Nearly all the tropical accessions (96.2\%) culstal together with indica accessions other than temperate japonica accessions in the phylogenetic tree constructed with the variants in TT1 located blocks. However, only a few indica accessions (5\%) cluster together with the tropical japonica. GLW7, which is a grain size related gene, is found to have great $F_{s t}$ between the temperate japonica and indica. However, significantly weaker $F_{s t}$ was found between the tropical japonica and indica in GLW7 gene region. By checking the $1 \mathrm{Mb}$ sequence flanking the both sides of GLW7 region, we find that the tropical accessions that exist introgression have identical haplotype with indica accessions (Figure S2). The phylogenetic tree constructed with the genetic variants in the flanking regions shows that the divergence patterns of different groups confers well with the haplotype map distribution. So our phylogenetic tree method detects the genetic introgression within the rice groups with great accuracy.

\section{Characterization of the introgression within the cultivated rice with the calibration of the wild rice}


There may be existence of errors to detect the genetic introgression regions with only the phylogenetic tree method, so we also apply another different D-static method to characterize genetic introgression within different groups to make a comparation. We have found more genetic introgression between tropical japonica and indica with the phylogenetic tree method, which may be caused by the overlap of their distribution areas (South side of East Asia). Previous reports have found that the domestication of Asia cultivated rice population is single origin, some genome segments are conserved within different cultivated rice groups. As the conserved segments will may disturb the phylogenetic tree analysis, D-static is a useful way to calibrate these effects by using the outgroup species as the background. As our study has found extensive introgression between the tropical japonica and indica, we decide to focus on the introgression between the two groups here (Figure 4). The detailed settings for the four groups used by the D-static calculation are illustrated in the methods (Wang et al., 2014). We have also tried to use the wild rice as outgroup to calculate the D-static value for the cultivated rice group. However, it results in too much introgression regions across the genome. We think that the extensive introgression detected by using the wild rice as the outgroup may be caused by the conserved genome segments of wild rice in the cultivated rice. The African rice is reported to have great genetic distance with the Asian cultivated rice. Only few genetic introgression regions are detected between the African cultivated rice and Asian cultivated rice in the previous study. So we set the African cultivated rice as outgroup in the D-static calculation.

After calculating the D-static value between the tropical japonica and indica population, we also find enrichment of genetic introgression in some regions of rice genome. Some high introgression blocks detected in the phylogenetic tree method overlap with the introgression peaks detected in the D-static method. There is also excessive high genetic introgression in some known introgression gene regions. The highest introgression peak in chromosome 3 contains the known heat resistance gene TT1, the genetic introgression peak of chromosome 7 contains the known gene GLW7 (Figure 4). For the high genetic introgression regions from tropical japonica to the indica, nearly no genetic introgression is detected in the direction from indica from to the tropical japonica. So it again proves that the detected genetic introgression is unidirectional, which is consistent with the results detected using the phylogenetic method. Different from the phylogenetic method the genetic introgression region detected in the D-static method is wider than those detected in the phylogenetic method. As similar wide genetic introgression regions are also detected in the other reports using the D-static method, this wide introgression region may be caused by the algorithm of defining the introgression region. In total the two methods get consistent results in the detection of genetic introgression region.

\section{Common introgression regions detected within the different cultivated rice subgroups}

After conducting analysis of genetic introgression regions within different rice subgroups, we find consistent high genetic introgression in a $4 \mathrm{Mb}$ region $(9.5 \mathrm{Mb}-13.5 \mathrm{Mb})$ near the centromere of chromosome 5(Table S2). It's found to be a high sequence repeat region from the annotation of Nipponbare reference genome (International Rice Genome Sequence Project. 2005). Previous report has 
found this region to be under strong selective pressure (Huang et al., 2012). We want to know whether the consensus high introgression region is the conserved segement of the ancient wild rice species or introgression from other cultivate rice populations. The japonica population is found to be domesticated from the Or-III wild rice group. The indica population is domesticated from the Or-I wild rice group. By using the construction of phylogenetic tree method we characterize the genetic introgression between the Or-I wild rice group and the indica population. The genetic introgression between the Or-III group and japonica population is also analyzed using the same method. It's found to have high genetic introgression between the Or-I wild rice group and the indica population. However, few genetic introgression accessions are detected between the japonica population and Or-III wild rice group. To further dissect the evolutionary relationship of this consensus introgression region, we construct phylogenetic tree with the genetic variants located in the region from $9.5 \mathrm{Mb}$ to $13.5 \mathrm{Mb}$ using the Oryza meridionallis as the outgroup (Figure 5). The phylogenetic tree shows that all the cultivated rice accessions are found to cluster together in a small region, which is consistent with the previous result of low genetic diversity in the cultivated rice. Though the cultivated rice is generally divided into the indica subgroup and japonica subgroup, we find mixture of some rice accessions between the two groups in the constructed phylogenetic tree. The Or-III wild rice subgroup has the closest relationship to the cultivated rice among all the wild rice populations. Interestingly, Or-I wild rice subgroup was found to have the greatest distance to cultivated rice accessions. However, these results are different from the phylogenetic tree constructed with the whole genome variation data, which shows divergence of indica and japonica subgroups. The Or-I and Or-III wild rice subgroup cluster together with the two different cultivated rice subgroups respectively. It may suggest closer relationship for Or-III with the indica and japonica population in this consistent introgression region.

Besides the phylogenetic tree, genetic diversity between different groups can also be used to dissect the relationship of different populations. We decide to study the genetic diversity in different rice groups to track the origin of this region. As we find different genetic diversity in the three big rice groups (indica, japonica and wild rice), we decide to characterize the whole genome genetic diversity in small groups of our collected materials to make a detailed comparison. All the small groups of wild rice have high genetic diversity in the region from $9.5 \mathrm{Mb}$ to $13.5 \mathrm{Mb}$, while all the cultivated rice small groups have low genetic diversity in this region except the Aus cultivated group. Aus is found to originate from the cross of the Or-I wild rice group and Ancient japonica in the previous report, which is similar to the indica population. So the origin of the consensus introgression in the cultivated rice may be from the Or-III group. It's under high human selection pressure, which is conserved in all the cultivated rice population in rice domestication process. However, in the formation of indica and Aus population, there is introgression of some genome segments from the Or-I group to the indica population and Aus population. As most of Aus accessions are found to cluster with the Or-I group other than indica and japonica population in the phylogenetic tree analysis (Figure 5), it suggests more genetic introgression from the Or-I wild rice group in Aus population than the indica population.

\section{Characterization of the biological functions for the introgression region using admixture mapping method}


Admixture association mapping studies had been reported in many species, such as human, dog, maize and so on. It's often used to locate the loci that cause special phenotypes between different groups. The Africa American is a good human population for genetic association mapping between the black man population and the white man population (Reiner et al., 2005). Many admixture mapping association studies had been conducted in this population to study about the cardiovascular, body mass index and other diseases to locate disease related alleles.

Different from the whole genome association study, which associates the genotype with the phenotype, the genome components of different populations are used to be associated with the phenotype to locate the phenotype related region in the admixture association mapping analysis. If there is no genetic introgression in the region that is associated with this phenotype, we can't locate the phenotype related region by admixture association mapping in a population even with big phenotype differences between the two subgroups. As there are few reports about admixture mapping association analysis in the crops, our study decides to characterize genetic admixture within the cultivated rice population. As our study has found some excessive genetic introgression loci between the indica population and japonica population, we decide to focus on the admixture association mapping between the indica and the two japonica subgroups (temperate japonica and tropical japonica). We conduct admixture association mapping with 11 previously published Asia cultivated rice agronomic traits (Figure 6, Figure S4-S14). Many phenotype related regions, which are detected in the admixture association mapping, are also reported in the previous genome wide association studies using the same phenotype. Some known phenotype related genes are located in the peak association regions of admixture mapping. For example, there are GS3, AIK, Waxy and so on (Sweeney et al., 2006, Wan et al, 2006, Sano et al, 1984,Saitoh et al., 2004,Li et al., 2015,Gao et al., 2003,Fan et al., 2006). So we can find that many phenotype associated regions detected in GWAS exists genetic introgression in the small blocks between indica and japonica(Figure S15-16). In this study we take the amylose content and grain length as an example to explain the results of admixture mapping.

There are two evident association peaks in the admixture mapping association results between the indica and tropical japonica population for the amylase content phenotype. There are also two association peaks in the same region in previous genome association study with the same trait. Two known phenotype related genes Waxy and $A L K$ are found to be located around the peaks (Figure S7A-D). The two association peaks can only be detected by GWAS in the indica population and whole population. However, they can't be detected by GWAS in japonica population for the low their genetic diversity. Similar results are also found in admixture association mapping results. Strong sharp peaks are detected in the association results between the indica and tropical japonica population. However, moderate peaks are detected in the association results between the indica and temperate japonica population. There are two different alleles for the Waxy gene in the rice genome, they are $W x^{a}$ and $W x^{b}$. The $W x^{a}$ type is an ancient type which exists in the wild rice population and indica population. $W x^{b}$ is a variant type which exists in the japonica population. So our admixture mapping results show that there is genetic introgression 
between the indica population and tropical japonica population in our collected materials. These genetic introgression regions between different groups are also validated by our phylogentic tree method.

Similar results are found in the grain length phenotype admixture association mapping results. There is no evident association peaks in the association results between the indica and temperate japonica population. However, strong association peaks are found in the results between the indica and tropical japonica population. The known grain length related gene GS3 is located in the association peaks of chromosome 3 (Figure 7E-H). GS3 was found to exist genetic introgression between the indica and tropical population in the previous study (Figure S3). The phenotype related regions detected by our admixture association mapping method are highly reliable introgression loci between different groups. Many phenotype related locus of rice subgroups may be introduced from other rice population by genetic introgression. Besides the overlapping of association peaks with the GWAS results, our study also detects many phenotype related regions that are missed by the GWAS method. These new phenotype related candidate regions can be further investigated by construction of related hybrid population to further study their biological functions.

\section{Discussion}

Genetic introgression is often used in human population to explore the genetic flow between populations. It can used to locate the alleles that are responsible for the population specific phenotypes (Durand et al., 2011, Reiner et al., 2005). There are also reports in crops, such as maize. They detect introgression between cultivated maize and wild maize (Hufford et al., 2013). Our study characterizes genetic introgression within cultivated rice subgroups in whole genome level with high density SNP markers. We get consistent results of genetic introgression regions between different cultivated rice groups with two different methods. Excessive genetic introgression is found between the indica population and tropical japonica population, which may be caused by the overlapping of distribution areas. They both have distribution in the south side of East Asia. So the tropical japonica may origin from temperate japonica by genetic introgression and forms a special ecotype after acquiring many alleles from the indica to adapt the local environment in south side of East Asia. For example, TT1 which enhances the heat resistance ability of tropical japonica is found to be located in the highest genetic introgression peaks between indica and tropical japonica (Li et al., 2015). By analyzing the flanking sequence around TT1 locus, we find that the tropical japonica has identical haplotypes with the indica population. Using admixture association mapping method we find that some agronomic traits related regions also exists moderate level of genetic introgression. They are also validated by the some known introgression genes (Waxy, ALK and GS3). Some admixture association peaks can only be detected between the indica and tropical population. It may suggest that the tropical japonica population get some phenotype related loci from the indica by genetic introgression.

The genetic region near the centromere of chromosome 5 was found to show high genetic introgression in different groups. By phylogenetic tree analysis we find that this region may origin from the Or-III wild rice group in the cultivated rice, as the Or-III has the closest relationship with the cultivated rice (Figure 5). 
After analyzing the genetic introgression between the cultivated rice and their related progenitors, we detect no genetic introgression between Or-III and the japonica, only moderate genetic introgression is found between the Or-I and indica. By analyzing the genetic diversity within the different groups of rice accessions, we find low genetic diversity in the whole cultivated rice except the Aus population. Most of the Aus accessions are found to cluster with Or-I group in phylogenetic tree constructed with the genetic variants located in the consensus high introgression region. So this region may origin from the Or-III group in the cultivated rice, it's conserved in the cultivated rice population after domestication selection. Some genome segments of the indica population are introduced to the tropical japonica and Aus population by genetic introgression from the Or-I group in the domestication process. Our study characterizes the genetic introgression within the different cultivated rice groups in the whole genome level. These introgression regions are annotated by admixture association mapping study, which provides good resource for the investigation of the gene function and genetic markers for the breeding process.

\section{Methods}

\section{Materials and phenotypes}

One part of the sequence data used in this study and whole phenotype data is from the previous studies (Huang et al., 2011, Huang et al., 2012). Their average sequence coverage is about 1 fold. We download 341 tropical japonica accessions and 199 Aus accession from the rice 3K genome project (Rellosa et al., 2014, Li et al., 2014 Wang et al., 2018). 88 African cultivated rice sequence data is also downloaded from the public database (Wang et al., 2014). Sratoolkit is used to convert the binary sra format to the text fastq format.

\section{Genotyping from the low coverage sequence data}

Smalt (version 0.75) software is used to align all the short sequence reads to the rice reference genome (IRGSP version 4) to generate the aligned cigar format. Self customized perl scripts are used to filter the aligned results. Only sequences that have unique match are conserved. The sequence alignments that exist low mapping score $(<60)$ and low mapping rate $(<82 \%$ of the total length)are filtered. We also exclude the sequence that has mismatch rate bigger than $8 \%$ of the total length. Using the id of the filtered sequence we retrieve the reads sequence from raw sequence data. Pileup software (version 0.4) is used to pileup the filtered reads to call SNP from the sequence data. For the detected single nucleotide polymorphism of the whole population we only keep the polymorphic sites with minor allele frequency bigger than $5 \%$ and missing rate smaller than $40 \%$.

\section{Characterization of genetic introgression using the phylogenetic tree method}

In our study the whole genome variation data of the cultivated rice is divided into $500 \mathrm{~kb}$ non-overlapping blocks. Using the self-customized C++ program, we generate kinship matrix for the population in the small block. The kinship matrix is converted to input format of phylip (version 3.69). The neighbor 
module of the phylip software is used to generate the phylogenetic tree for each small block. Phylogentic tree using the whole genome variation data is first used to determine the group for each accession. The ape package of the $\mathrm{R}$ language is used to present this phylogenetic tree and label them with different colors according to the subgroup they belong to. For the two groups that we want to characterize genetic introgression we set them as group $A$ and group $B$. Some accessions belonging to group $A$ in the whole genome level cluster with group $B$ in the small block. These accessions are found to have genetic introgression from group $B$ to group $A$ in this block.

\section{Characterization of genetic introgression with D-static value}

Our study also use the previous reported "ABBA-BABA" method (D-static method) to characterize the genetic introgression (Durand et al., 2011, Zhang et al., 2016). It needs four populations to calculate the $D$-static value. We label them as $\{P 1, P 2, P 3,0\}$. P1 and $P 3$ are the groups that we want to detect genetic introgression. P2 is an ancient population that is similar to P1 and P3. $\mathrm{O}$ is the ancient outgroup. We calculate the allele frequency for the four different groups. The following equation is used to calculate the D-static value (Zhang et al., 2016).

$\mathrm{D}(\mathrm{P} 1, \mathrm{P} 2, \mathrm{P} 3, \mathrm{D})=\frac{\sum_{\mathrm{i}=1}^{\mathrm{n}}\left[\left(1-\mathrm{p}_{\mathrm{i} 1}\right) \mathrm{p}_{\mathrm{i} 2} \mathrm{p}_{\mathrm{i} 2}\left(1-\mathrm{p}_{\mathrm{i} 4}\right)-\mathrm{p}_{\mathrm{i} 1}\left(1-\mathrm{p}_{\mathrm{i} 2}\right) \mathrm{p}_{\mathrm{i} 3}\left(1-\mathrm{p}_{\mathrm{i} 4}\right)\right.}{\sum_{\mathrm{i}=1}^{\mathrm{n}}\left[\left(1-\mathrm{p}_{\mathrm{i} 1}\right) \mathrm{p}_{\mathrm{i} 2} \mathrm{p}_{\mathrm{i} 2}\left(1-\mathrm{p}_{\mathrm{i} 4}\right)+\mathrm{p}_{\mathrm{i} 1}\left(1-\mathrm{p}_{\mathrm{i} 2}\right) \mathrm{p}_{\mathrm{i} 3}\left(1-\mathrm{p}_{\mathrm{i} 4}\right)\right.}$

In our study we set tropical japonica as P1, temperate japonica as P2, indica as P3, African cultivated rice as the 0 . The equation above is used to calculate the $\mathrm{D}$-static value.

\section{Admixture association mapping with 11 agronomic traits}

The whole genome variation data is sliced into $200 \mathrm{~kb}$ non-overlapping blocks. The genetic variants are converted to the ped and map format for Admixture (Alexander et al., 2009) using self-customized perl scripts. The genome component for the different groups ais determined for all the accessions. The genome components matrix of the whole population is used to associate with phenotype using mixed linear model to generate the final association results.

\section{Declarations}

\section{Acknowledgements}

This work was partially supported by The Professorial and Doctoral Scientific Research Foundation of Huizhou University(2020JB068).

\section{Availability of data}

The datasets supporting the conclusions of this article are included within the article and its additional files. The cultivated rice sequence data was downloaded from the EBI European Nucleotide Archive with 
accession number ERP000729 and ERP000106. The data for the wild rice accession was downloaded from the EBI with ERP001143, ERP000729 and ERP000106.

\section{Authors' contributions}

B.H. conceived the project. H.G. and B.H. designed and supervised the project and wrote the manuscript. H.G. performed most of the data analysis.

\section{References}

1. Alexander DH, Novembre J, Lange K. Fast model-based estimation of ancestry in unrelated individuals. Genome Res. 2009;19:1655-64.

2. Ando T, Yamamoto T, Shimizu T, Ma XF, Shomura A, Takeuchi Y, et al. Genetic dissection and pyramiding of quantitative traits for panicle architecture by using chromosomal segment substitution lines in rice. Theor Appl Genet. 2008;116:881-90.

3. Chen Z, Li X, Lu H, Gao Q, Du H, Peng H, et al. Genomic atlases of introgression and differentiation reveal breeding footprints in Chinese cultivated rice. J Genet Genomics. 2020;47:637-49.

4. Civáň $P$, Brown TA. Role of genetic introgression during the evolution of cultivated rice (Oryza sativa L.). BMC Evol Biol. 2018;18:57.

5. Durand EY, Patterson N, Reich D, Slatkin M. Testing for ancient admixture between closely related populations. Mol Biol Evol. 2011;28:2239-52.

6. Fan C, Xing Y, Mao H, Lu T, Han B, Xu C, et al. GS3, a major QTL for grain length and weight and minor QTL for grain width and thickness in rice, encodes a putative transmembrane protein. Theor Appl Genet. 2006;112:1164-71.

7. Gao Z, Zeng D, Cui X, Zhou Y, Yan M, Huang D, et al. Map-based cloning of the ALK gene, which controls the gelatinization temperature of rice. Sci China Ser C, Life Sci. 2003;46:661-8.

8. Giuffra E, Kijas JM, Amarger V, Carlborg O, Jeon JT, Andersson L. The origin of the domestic pig: independent domestication and subsequent introgression. Genetics. 2000;154:1785-91.

9. Heliconius Genome Consortium. Butterfly genome reveals promiscuous exchange of mimicry adaptations among species. Nature. 2012;487:94-8.

10. Huang X, Kurata N, Wei X, Wang Z-X, Wang A, Zhao Q, et al. A map of rice genome variation reveals the origin of cultivated rice. Nature. 2012;490:497-501.

11. Huang X, Zhao Y, Wei X, Li C, Wang A, Zhao Q, et al. Genome-wide association study of flowering time and grain yield traits in a worldwide collection of rice germplasm. Nat Genet. 2011;44:32-9.

12. Huerta-Sánchez E, Jin X, Asan, Bianba Z, Peter BM, Vinckenbosch N, et al. Altitude adaptation in Tibetans caused by introgression of Denisovan-like DNA. Nature. 2014;512:194-7.

13. Hufford MB, Lubinksy P, Pyhäjärvi T, Devengenzo MT, Ellstrand NC, Ross-lbarra J. The genomic signature of crop-wild introgression in maize. PLoS Genet. 2013;9:e1003477. 
14. Huson DH, Richter DC, Rausch C, Dezulian T, Franz M, Rupp R. Dendroscope: An interactive viewer for large phylogenetic trees. BMC Bioinformatics. 2007;8:460.

15. Huson DH, Scornavacca C. Dendroscope 3: an interactive tool for rooted phylogenetic trees and networks. Syst Biol. 2012;61:1061-7.

16. Li X-M, Chao D-Y, Wu Y, Huang X, Chen K, Cui L-G, et al. Natural alleles of a proteasome a2 subunit gene contribute to thermotolerance and adaptation of African rice. Nat Genet. 2015;47:827-33.

17. Lin Z, Qin P, Zhang X, Fu C, Deng H, Fu X, et al. Divergent selection and genetic introgression shape the genome landscape of heterosis in hybrid rice. Proc Natl Acad Sci U S A. 2020;117:4623-31.

18. Molina J, Sikora M, Garud N, Flowers JM, Rubinstein S, Reynolds A, et al. Molecular evidence for a single evolutionary origin of domesticated rice. Proc Natl Acad Sci U S A. 2011;108:8351-6.

19. Okagaki RJ. Nucleotide sequence of a long cDNA from the rice waxy gene. Plant Mol Biol. 1992;19:513-6.

20. Racimo F, Sankararaman S, Nielsen R, Huerta-Sánchez E. Evidence for archaic adaptive introgression in humans. Nat Rev Genet. 2015;16:359-71.

21. Reiner AP, Ziv E, Lind DL, Nievergelt CM, Schork NJ, Cummings SR, et al. Population structure, admixture, and aging-related phenotypes in African American adults: the Cardiovascular Health Study. Am J Hum Genet. 2005;76:463-77.

22. Saitoh K, Onishi K, Mikami I, Thidar K, Sano Y. Allelic diversification at the C (OsC1) locus of wild and cultivated rice: nucleotide changes associated with phenotypes. Genetics. 2004;168:997-1007.

23. Sano Y. Differential regulation of waxy gene expression in rice endosperm. Theor Appl Genet. 1984;68:467-73.

24. Santos JD, Chebotarov D, McNally KL, Bartholomé J, Droc G, Billot C, et al. Fine Scale Genomic Signals of Admixture and Alien Introgression among Asian Rice Landraces. Genome Biol Evol. 2019;11:1358-73.

25. Sweeney MT, Thomson MJ, Pfeil BE, McCouch S. Caught red-handed: Rc encodes a basic helix-loophelix protein conditioning red pericarp in rice. Plant Cell. 2006;18:283-94.

26. Wan L, Zha W, Cheng X, Liu C, Lv L, Liu C, et al. A rice $\beta$-1,3-glucanase gene Osg1 is required for callose degradation in pollen development. Planta. 2011;233:309-23.

27. Wang M, Yu Y, Haberer G, Marri PR, Fan C, Goicoechea JL, et al. The genome sequence of African rice (Oryza glaberrima) and evidence for independent domestication. Nat Genet. 2014;46:982-8.

28. Wang W, Mauleon R, Hu Z, Chebotarov D, Tai S, Wu Z, et al. Genomic variation in 3,010 diverse accessions of Asian cultivated rice. Nature. 2018;557:43-9.

29. Wang ZY, Wu ZL, Xing YY, Zheng FG, Guo XL, Zhang WG, et al. Nucleotide sequence of rice waxy gene. Nucleic Acids Res. 1990;18:5898.

30. Wedger MJ, Pusadee T, Wongtamee A, Olsen KM. Discordant Patterns of Introgression Suggest Historical Gene Flow into Thai Weedy Rice from Domesticated and Wild Relatives. J Hered. 2019;110:601-9. 
31. Zhang W, Dasmahapatra KK, Mallet J, Moreira GRP, Kronforst MR. Genome-wide introgression among distantly related Heliconius butterfly species. Genome Biol. 2016;17:25.

\section{Figures}

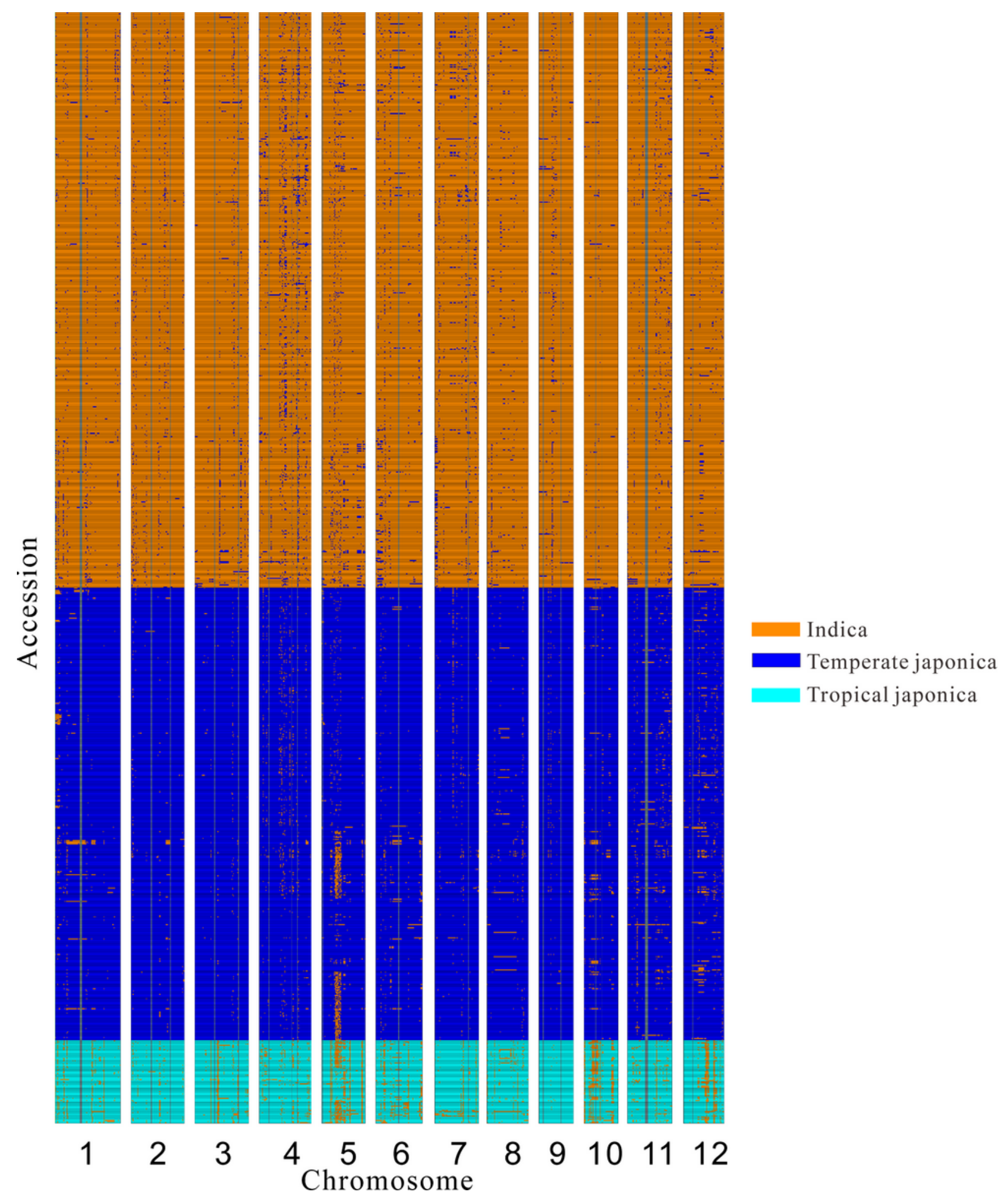

Figure 1 
Diagram for the genetic introgression in the major three subgroups of Asia cultivate rice. The horizontal line represents 12 chromosomes of the rice genome. The vertical line represents accessions in three groups. Each horizontal small line represents one accession of the cultivated rice population. The small rectangle on the map represents a small block for one accession. Different colors represent different genome components, the gray area represents genetic areas that have insufficient polymorphic sties to construct phylogentic tree.
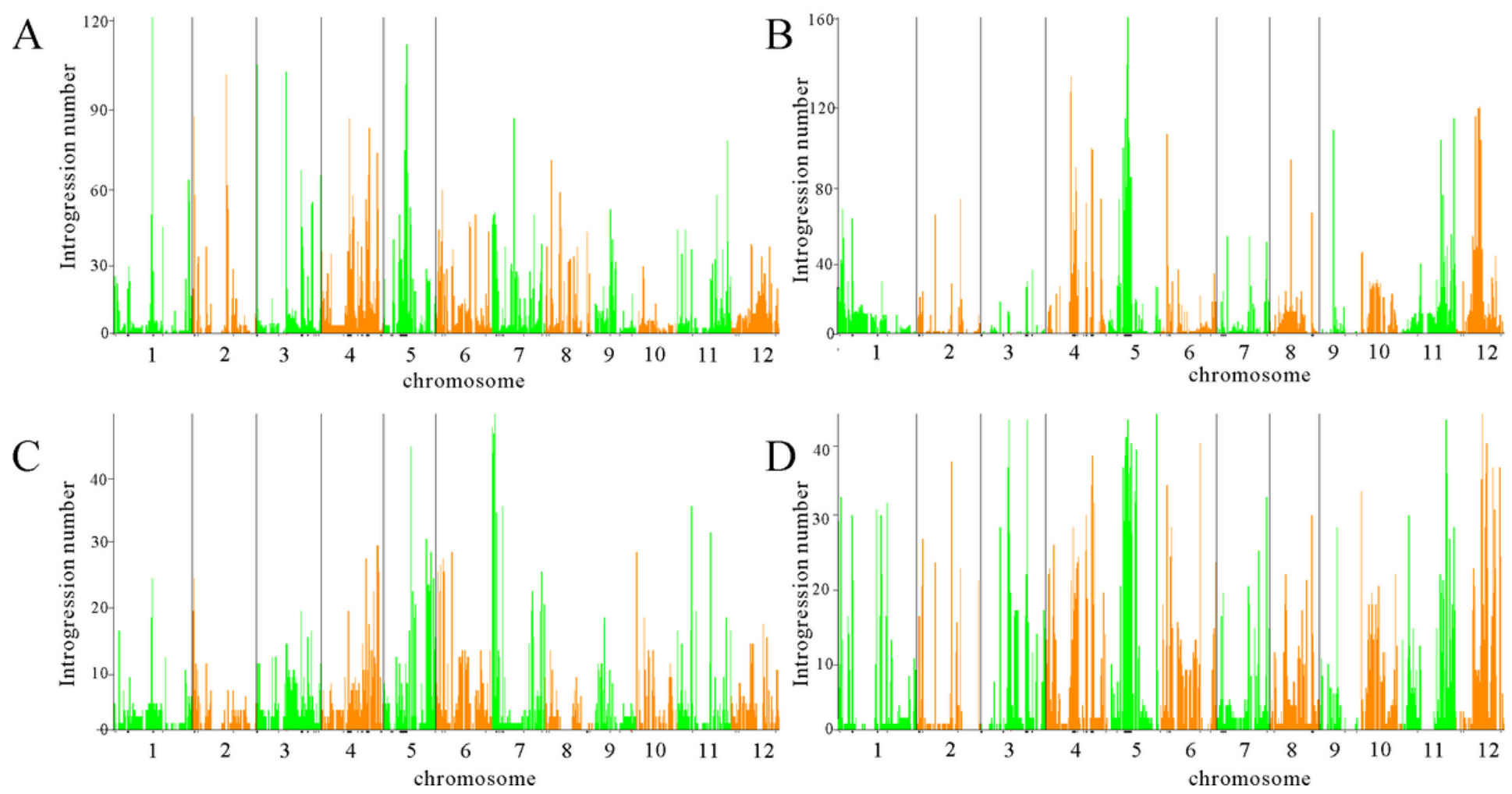

\section{Figure 2}

Summary of the cultivated rice accessions that exist introgression between different groups. The horizontal line for each diagram represents 12 chromosomes of the whole rice genome. The horizontal line represents the number of accession that has genetic introgression between different groups. The black line under the horizontal line represents the 55 major selective sweeps detected by the previous study. Figure A represents summary data for genetic introgression from the temperate japonica subgroup to the indica subgroup. Figure B represents the summary data for the genetic introgression from the indica subgroup to temperate japonica subgroup. Figure $C$ represents the summary data for the genetic introgression from tropical japonica subgroup to the indica subgroup. Figure $D$ represents the summary data for the genetic introgression from the indica subgroup to tropical japonica subgroup. 

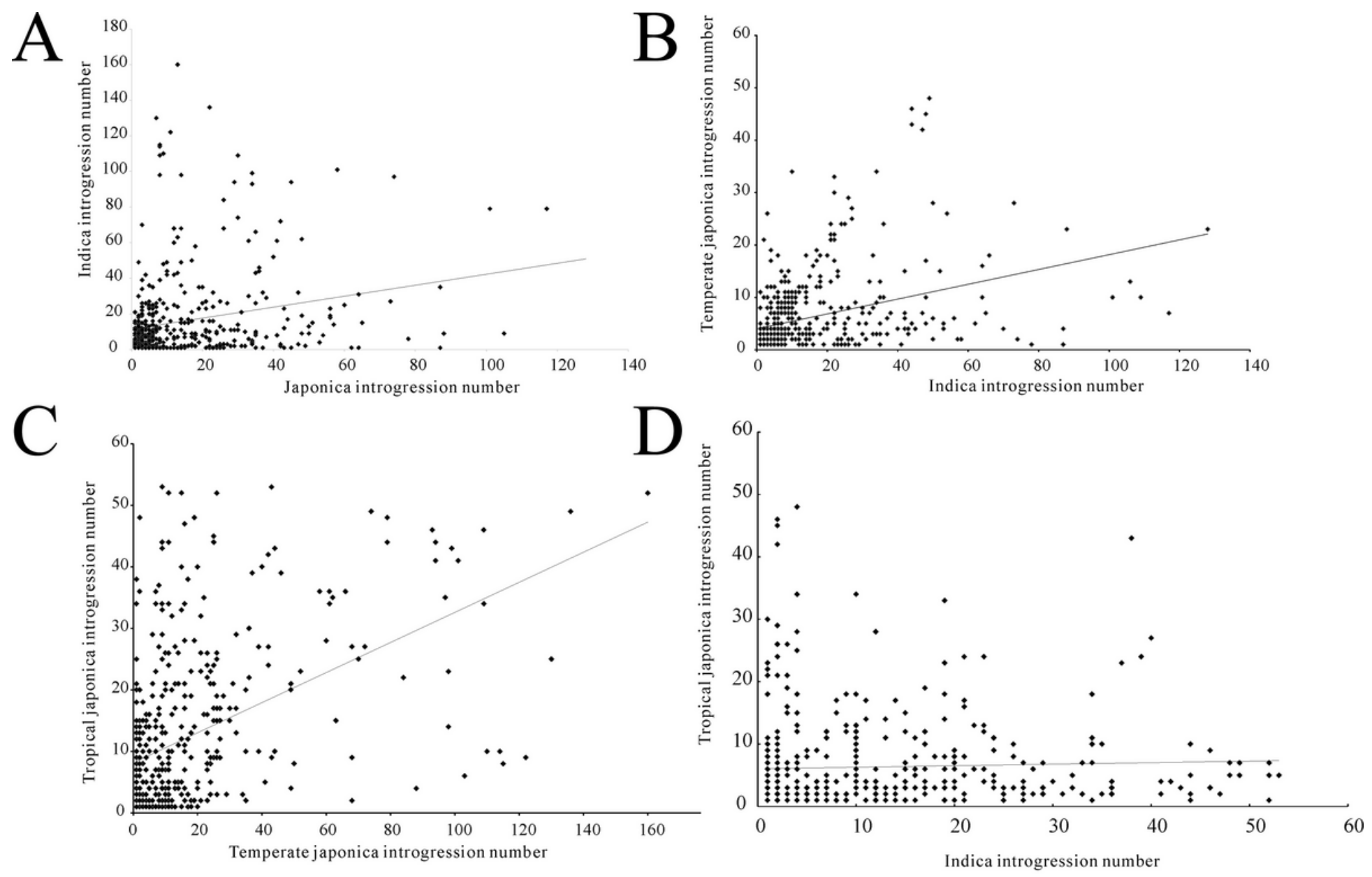

\section{Figure 3}

Correlation of introgression accession number between different subgroups. The horizontal line and vertical line represent different introgression number for different groups. The black line on the figure represents the regression line of the introgression data. The groups they represent on each line are labeled in the figure.

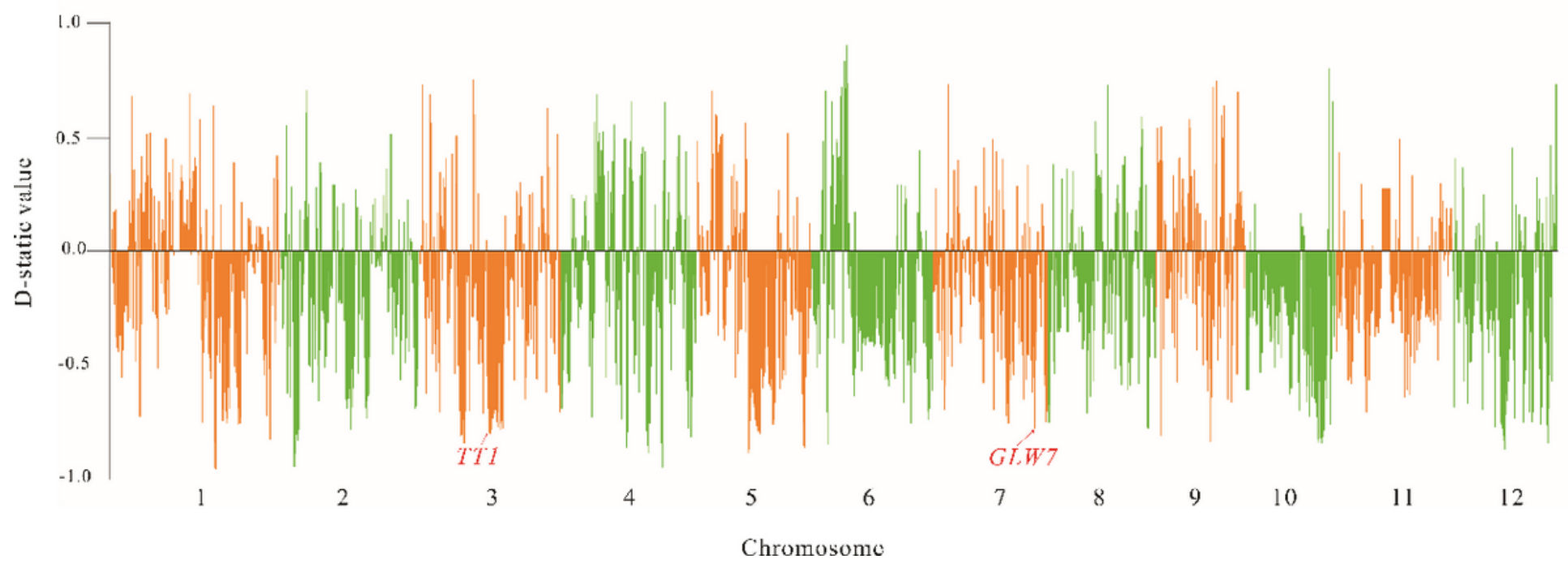

Figure 4 
Genetic introgression between the indica and tropical japonica population detected by the D-static value method. The horizontal line represents 12 chromosomes of the rice genome. The vertical line is the Dstatic value of the two groups. TT1 and GLW7 are two known genes that exists genetic introgression between the tropical japonica and indica population.

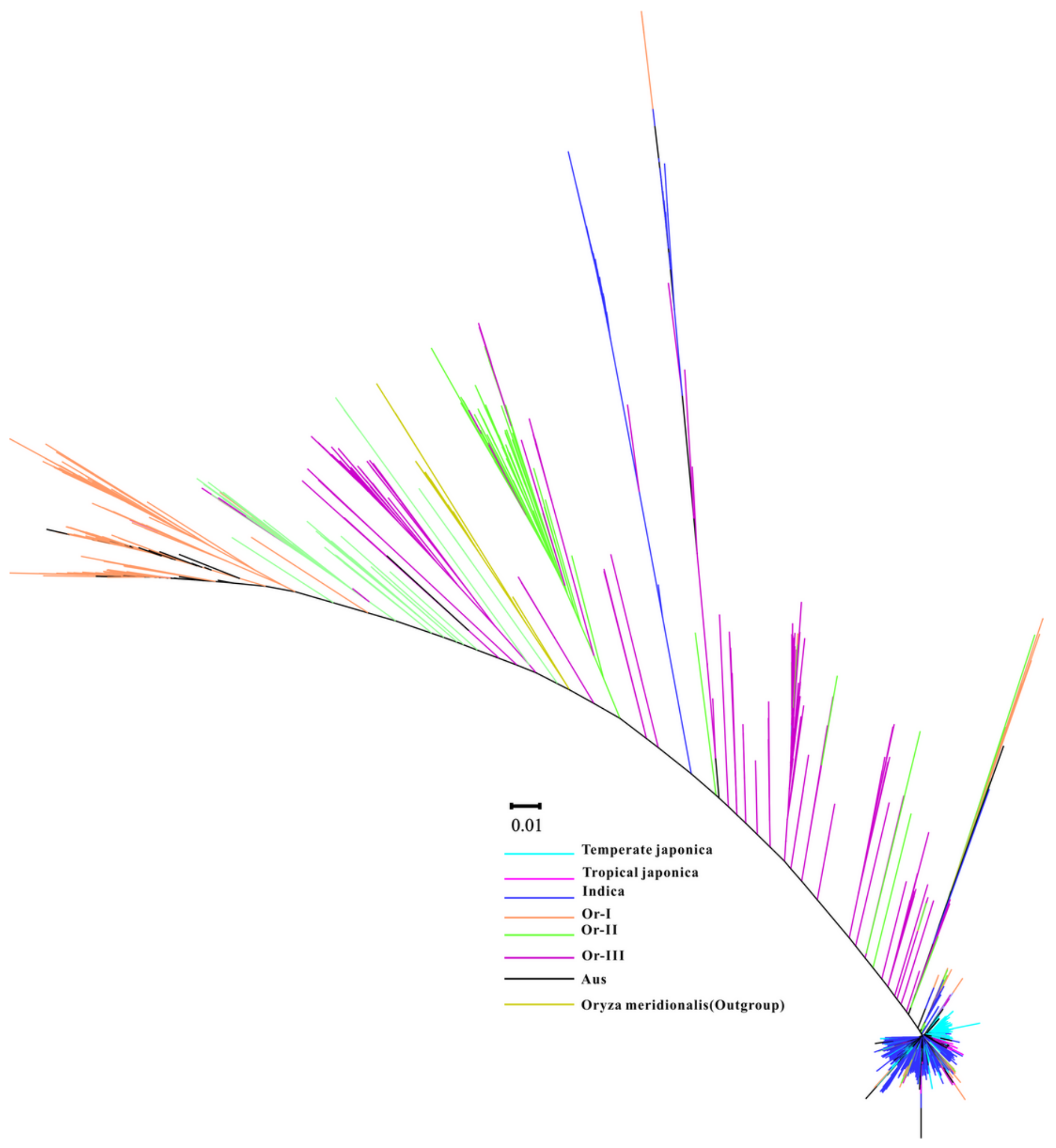

Figure 5 
the neighbor joining tree constructed with the genetic variants located in the centromere of chromosome 5. The genetic variants located in the region from $9.5 \mathrm{Mb}$ to $13.5 \mathrm{Mb}$ are used to construct the phylogenetic tree. Each subgroup of the wild rice is labeled with different color.

A Accession 1

Accession 2

Accession 3

Accession 4

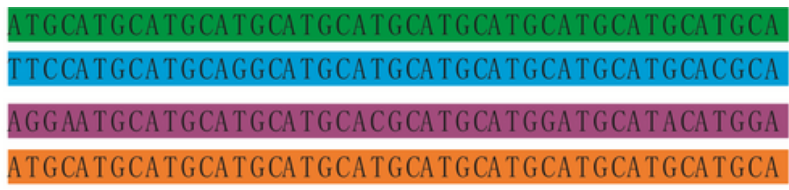

represents one ancestry

represents another ancestry

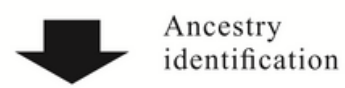

B Accession 1 Accession 2

Accession 3

Accession 4

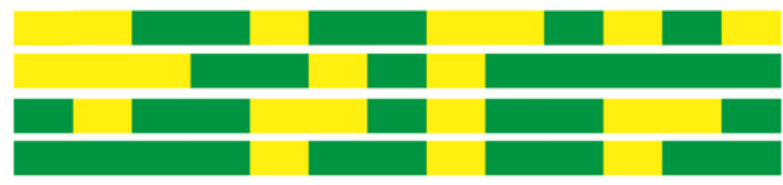

Ancestry matrix

C Phenotype 1 001101011011011011010101101101011100110110110 Phenotype 2001101011011011011010101101101011100110110110 Phenotype 3001101011011011011010101101101011100110110110

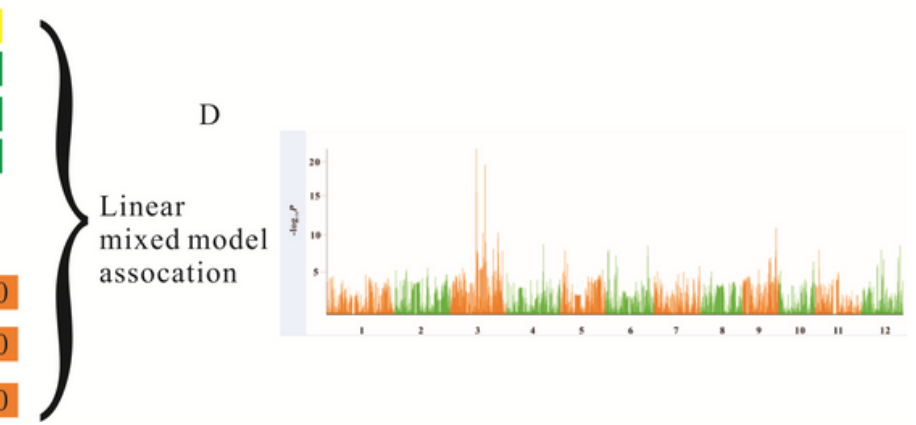

\section{Figure 6}

Work flow for the Admxiture association mapping in the cultivated rice. Figure A shows the haplotype map of four rice accessions. Figure $B$ shows the genome component detected by the Admixture software. The green color and yellow color represent the two different genome components. Figure $\mathrm{C}$ represents the phenotype data for different accessions. Figure $D$ shows the Admixture mapping results we get by associating the Ancestry component matrix with the phenotype. 


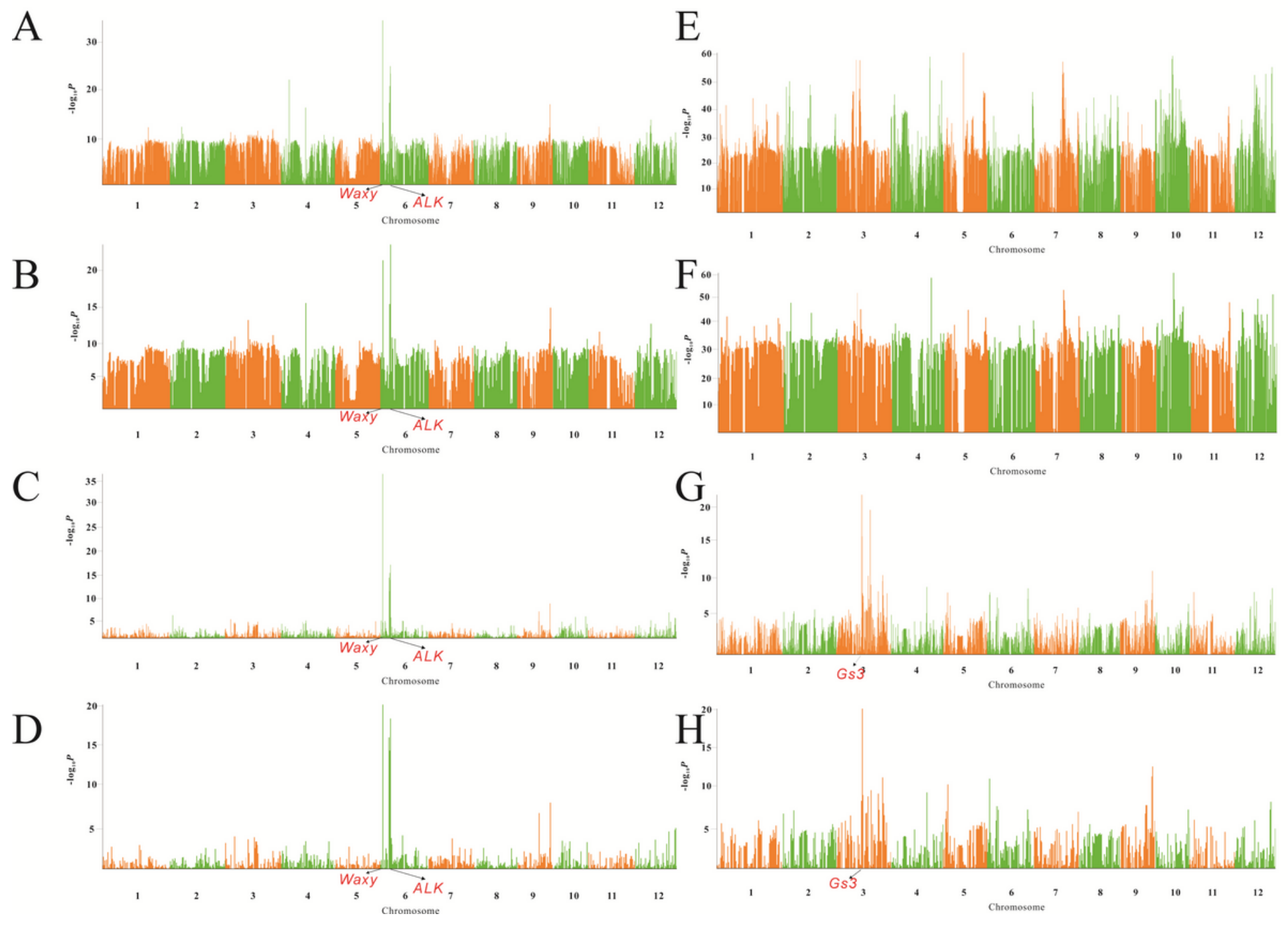

Figure 7

Admixture association mapping results using amylose content phenotype and the grain length phenotype. Figure A-D show the admixture association mapping results for the amylose content phenotype. Figure $\mathrm{E}-\mathrm{H}$ show the association results for the grain length phenotype. Figure $\mathrm{A}$ shows association result of the indica component detected in the merged population of indica and temperate population. Figure $E$ shows the result of grain length phenotype with the same data. Figure B shows association result of temperate japonica component detected in the merged population of temperate japonica and indica. Figure $\mathrm{F}$ shows the result of grain length phenotype with the same data. Figure $\mathrm{C}$ shows association result of the tropical japonica component detected in the merged population of tropical japonica and indica. Figure $\mathrm{G}$ shows association of grain length phenotype using the same data. Figure $D$ shows association result of the indica component detected in the merged population of tropical japonica and indica. Figure $\mathrm{H}$ shows the association result of grain length using the same data.

\section{Supplementary Files}

This is a list of supplementary files associated with this preprint. Click to download. 
- Supplementaryfile.docx

Page 19/19 